\title{
Multiobjective VLSI Cell Placement using Distributed Genetic Algorithm
}

\author{
Sadiq M. Sait Mohammed Faheemuddin Mahmood R. Minhas Syed Sanaullah \\ College of Computer Sciences \& Engineering \\ King Fahd University of Petroleum \& Minerals \\ Dhahran, Saudi Arabia
}

\{sadiq,faheem,minhas,sanaulla\}@ccse.kfupm.edu.sa

\begin{abstract}
Genetic Algorithms have worked fairly well for the VLSI cell placement problem, albeit with significant run times. Two parallel models for GA are presented for VLSI cell placement where the objectives are optimizing power dissipation, timing performance and interconnect wirelength, while layout width is a constraint. A Master-Slave approach is mentioned wherein both fitness calculation and crossover mechanism are distributed among slaves. A Multi-Deme parallel GA is also presented in which each processor works independently on an allocated subpopulation followed by information exchange through migration of chromosomes. A pseudo-diversity approach is taken, wherein similar solutions with the same overall cost values are not permitted in the population at any given time. A series of experiments are performed on ISCAS-85/89 benchmarks to show the performance of the Multi-Deme approach.
\end{abstract}

\section{Categories and Subject Descriptors}

D.1.3 [Programming Techniques]: Concurrent Programming-Parallel programming

\section{General Terms}

Algorithm, Performance

\section{Keywords}

Parallel Genetic Algorithms, Cluster Computing, Fuzzy Logic, Genetic Crossover.

\section{INTRODUCTION}

Genetic Algorithms have been extensively used for solving NP-hard problems such as VLSI cell placement $[2,3]$. Parallelization of these algorithms has increasingly become an attractive option for accelerating performance, especially due to the consistent growth in performance to cost ratios

Copyright is held by the author/owner.

GECCO'05, June 25-29, 2005, Washington, DC, USA.

ACM 1-59593-010-8/05/0006. of cluster computing environments. Two parallel GA strategies are described here that target the optimization of widthconstrained, multi-objective placement.

\section{PARALLELIZATION OF GENETIC ALGORITHMS}

Prior to developing parallelization strategies, a profiling analysis of the serial GA was carried out to determine computation intensive functions and routines. It was seen that the runtime intensive operations are fitness calculation and the parent crossover. The first parallel model is a 'Global Selection' approach where the population is divided among slave processors, which then carry out individual crossover followed by fitness evaluation of generated offsprings. Selection of the new population is done at the Master, which collects the cumulative offsprings from the slave processors. The performance results of this approach were poor with very low speedup for small circuits. However, with increasing circuit size, which translates into higher complexity and larger search space, there is more potential with distributing the fitness calculation and the crossover. In the case of smaller circuits, any gains achieved by such a distribution would be lost due to communication overheads.

The second approach - the Multi-Deme parallel GA, has often been favored over simplistic data distribution as in the earlier model. In this strategy, the population is distributed among all processors, which independently run their own GA for a predefined number of generations. An extensive study of the parameters governing the performance of this model was done by Cantú-Paz [1]. The pseudo-code of the algorithm is presented in Figure 1.

The initial population constructor runs on the master processor, which then distributes the solutions among all the slave processors. Following this, all nodes, including the root execute the serial GA on their allocated population for a predefined number of iterations called the Migration Frequency $(M F)$. Then each node sends a certain number of its best solutions to the root. The number of solutions sent is controlled by the Migration Rate $(M R)$ parameter. The root determines the $M R$ best solutions from the collective $M R *(N)$ solutions and broadcasts it to all processors. These migrants if not already present on the processors, are then absorbed into the existing population by weeding out and replacing the weakest solutions. Each processor then continues with the serial GA for another $M F$ number of generations. Every interval between migrations, i.e., the length of time defined by MF number of generations is called 
as Epoch. The stopping criteria is a predefined number of Epochs.

It is important to note that the migrant absorption policy dictates the replacement of worst solutions with incoming migrants only if the migrants already do not exist within the population. Also, logically this model could represent a fully connected topology of non-hierarchical processing elements which cooperate to determine the best $M R$ solutions among themselves and absorb these into their existing populations.

\section{RESULTS AND DISCUSSION}

The parallel architecture used in this work is a dedicated eight-node cluster connected via a low-latency network. Each of these nodes is a general purpose stand-alone Pentium4 workstation running at $2.0 \mathrm{GHz}$ with $256 \mathrm{MB}$ memory and running the RedHat Linux distribution. The cluster runs over a Fast-Ethernet switch. Communication between nodes is achieved using the MPICH implementation of the Message Passing Interface.

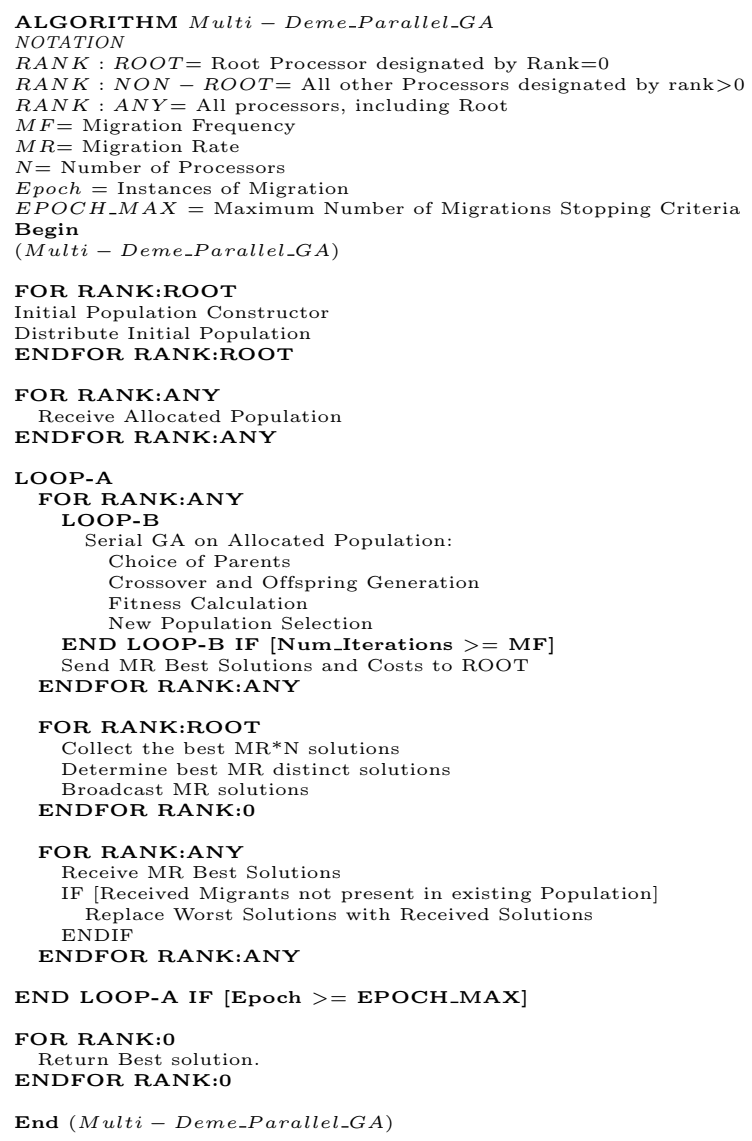

Figure 1: Structure of the Multi-Deme Parallel GA.

Results for the Multi-Deme Parallel GA are documented in Table 1. The Migration Frequency and Migration Rate are twenty and one respectively, i.e., all processors run the GA on their allocated sub-population for twenty generations, followed by migration of one chromosome between them. The GA parameters are the same as used for the serial Genetic Algorithm. Figure 2 gives the speedup performance for the s386 circuit.
Table 1: Multi-Deme Parallel GA: Runtime to reach a target fitness with increasing number of processors.

\begin{tabular}{|c|c|c|c|c|c|c|c|c|c|}
\hline Circuit & Target & \multicolumn{70}{|c|}{ Time taken to reach target fitness } \\
\cline { 3 - 11 } & Fitness & $\mathrm{P}=1$ & $\mathrm{P}=2$ & $\mathrm{P}=3$ & $\mathrm{P}=4$ & $\mathrm{P}=5$ & $\mathrm{P}=6$ & $\mathrm{P}=7$ & $\mathrm{P}=8$ \\
\hline \hline $\mathrm{s} 298$ & 0.73 & 219 & 116 & 79 & 62 & 48 & 42 & 37 & 36 \\
\hline $\mathrm{s} 386$ & 0.63 & 314 & 171 & 109 & 89 & 71 & 62 & 52 & 52 \\
\hline $\mathrm{s} 832$ & 0.54 & 569 & 306 & 199 & 155 & 122 & 105 & 89 & 88 \\
\hline $\mathrm{s} 953$ & 0.54 & 1004 & 549 & 354 & 280 & 222 & 191 & 162 & 162 \\
\hline $\mathrm{s} 641$ & 0.64 & 2734 & 1439 & 933 & 730 & 589 & 520 & 425 & 424 \\
\hline $\mathrm{s} 1196$ & 0.54 & 1538 & 876 & 549 & 439 & 348 & 299 & 247 & 248 \\
\hline $\mathrm{s} 1494$ & 0.53 & 1679 & 942 & 597 & 460 & 367 & 319 & 263 & 268 \\
\hline $\mathrm{s} 1488$ & 0.54 & 1672 & 913 & 592 & 459 & 368 & 316 & 266 & 268 \\
\hline $\mathrm{s} 3330$ & 0.50 & 6818 & 3959 & 2584 & 1933 & 1523 & 1317 & 1090 & 1094 \\
\hline
\end{tabular}

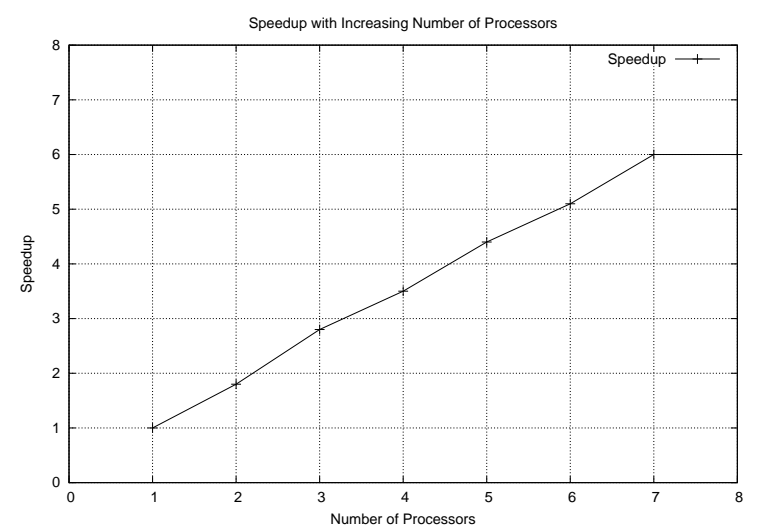

Figure 2: Speedup for circuit s386. The speedup pattern is almost identical for all circuits

\section{CONCLUSION}

This paper primarily serves as a demonstration of documented GA parallelization strategies to multiobjective optimization problems. The first approach was a variation of the canonical Master-Slave parallel GA, with both fitness and crossover distributed among processors. Only Selection was implemented by the Master. Performance gains in terms of reduced run-time were seen only for larger circuits. On the other hand, the Multi-Deme approach reported consistent performance gains independent of problem complexity and size of the search space.

\section{ACKNOWLEDGMENT:}

The authors acknowledge King Fahd University of Petroleum \& Minerals (KFUPM), Dhahran, Saudi Arabia, for support under Project Code COE/CELL PLACE/263.

\section{REFERENCES}

[1] E. Cantú-Paz. Designing Efficient Master-Slave Parallel Genetic Algorithms. Genetic Programming 1998: Proceedings of the Third Annual Conference, 1998.

[2] S. M. Sait and H. Youssef. Iterative Computer Algorithms and their Application to Engineering: Solving Combinatorial Optimization Problems. December 1999.

[3] S. M. Sait, H. Youssef, A. El-Maleh, and M. R. Minhas. Iterative Heuristics for Multiobjective VLSI Standard Cell Placement. Proceedings of IJCNN'01, International Joint Conference on Neural Networks, 3:2224-2229, July 2001. 